\title{
Radiation Responses of Hemopoietic Colony-Forming Cells Derived from Different Sources ${ }^{1}$
}

\author{
L. SIMINOVITCH, J. E. TILL, AND E. A. McCULLOCH \\ Department of Medical Biophysics, University of Toronto, and The Ontario Cancer \\ Institute, Toronto, Canada
}

\section{INTRODUCTION}

Evidence is accumulating $(1,2)$ that the regeneration of the hemopoietic system, and hence survival of the animal, after exposure to whole-body irradiation is due to the proliferation of a class of progenitor cells that possess the capacity for colony formation in the spleens of irradiated hosts. At the present time, such progenitor cells cannot be identified by histological techniques, nor have they yet been extensively purified (3). Thus, their only distinguishing characteristic is their ability to form spleen colonies. It is possible that this property might be shared by cells very different in other regards. Preliminary indications in favor of this view were provided by an earlier series of experiments (4) in which it was found that colonyforming cells derived from spleen colonies had a decreased capacity for self-renewal when compared with colony-forming cells derived from normal adult marrow. The fact that cells with colony-forming ability can be obtained not only from femoral marrow but also from adult spleen (5), peritoneal fluid and peripheral blood (6), fetal liver (5), and spleen colonies ( 7 ) suggested another way in which the homogeneity of homopoietic colony-forming cells might be tested, since the properties of colony-forming cells from these various sources might be compared.

In this paper, a comparison is made of the radiation survival curves obtained for the colony-forming ability of cells derived from femoral marrow, spleen, and fetal liver. The results indicate that the radiosensitivity of colony-forming ability can be dependent on the origin and previous history of the cells under test. This finding supports the view that hemopoietic colony-forming cells do not necessarily represent a homogeneous class of cells. On this basis, models of the effects of totalbody irradiation on the hemopoietic system which are based on the simplifying

1 This research was supported by the Defence Research Board, (Grant 9350-14 G and C), by the Medical Research Council (Grant MA-1420), by the National Cancer Institute of Canada, and by the National Research Council (Grant T-1714). 
assumption that colony-forming cells possess a characteristic, unique radiation sensitivity may have to be modified.

\section{MATERIALS AND METHODS}

\section{Mice}

The mice used in these experiments were $\mathrm{C} 57 \mathrm{BL}, \mathrm{C} 3 \mathrm{H}$, or the $\mathrm{F} 1$ hybrid between them. These mice were derived from two colonies maintained separately at the Ontario Cancer Institute. The designations of the strains used are: $\mathrm{C} 57 \mathrm{BL} / \mathrm{HaOci}$ and $\mathrm{C} 3 \mathrm{H} / \mathrm{He}_{\mathrm{f}} \mathrm{Oci}$ (main colony); $\mathrm{C} 57 \mathrm{BL} / 6 \mathrm{JOci}$ and $\mathrm{C} 3 \mathrm{H} / \mathrm{HeOci}$ (annex colony). In all experiments, isologous mice were used as donors and recipients of cell transplants.

\section{Survival Curve for Colony Formation}

Measurements of the colony-forming ability of suspensions of hemopoietic cells were carried out by using the spleen colony technique, as described previously $(8$, 9 ). Survival curves for hemopoietic cells were obtained by the in vivo irradiation technique (9), as follows: Mice to be used as recipients of transplants of hemopoietic cells were given a preliminary dose of X-rays (280 kvp, HVL $1.12 \mathrm{~mm} \mathrm{Cu}$, approximately $100 \mathrm{r} / \mathrm{min}$ ) and then injected with an appropriate aliquot of cells from the suspension under test. After a 2-hour interval, the animals bearing the transplanted cells were exposed to $\gamma$-rays of varying magnitude (dose rate approximately $85 \mathrm{rads} / \mathrm{min}$ ) from a $\mathrm{Co}^{60}$ source. The $\mathrm{X}$-ray and $\gamma$-ray doses were partitioned in such a way that the recipient mice received a total dose of 900 to 1000 rads, which has been shown to be large enough to suppress colony formation by endogenous cells (1); the injected cells were exposed only to the dose of $\gamma$-radiation.

Radiation dosimetry was carried out as described previously, with the Fricke ferrous sulfate dosimeter (10). A $G$ value of 15.5 ferric ions per $100 \mathrm{ev}$ was assumed.

\section{Preparation of Cell Suspensions}

The methods used for obtaining cell suspensions from femoral marrow (8), spleen and fetal liver (5), and spleen colonies ( 7$)$ have been described. In each case singlecell suspensions containing very few clumps were obtained.

In a number of experiments, cell suspensions were obtained from femoral marrows or spleens of irradiated animals (900 rads of X-rays, single exposure) bearing transplants derived from known numbers of marrow, spleen, or fetal liver cells. The yields of nucleated cells from the spleens and femora of such animals and the colonyforming efficiencies of the cell suspensions obtained from these sources, as well as those of cell suspensions obtained from normal, unirradiated donors, are given in Tables I and II. For example, from line 1 of Table I it may be seen that cell suspensions obtained from normal adult femoral marrow contained, on the basis of six experiments, a mean of $3.3 \times 10^{7}$ cells per two femora, and that the colony-forming 


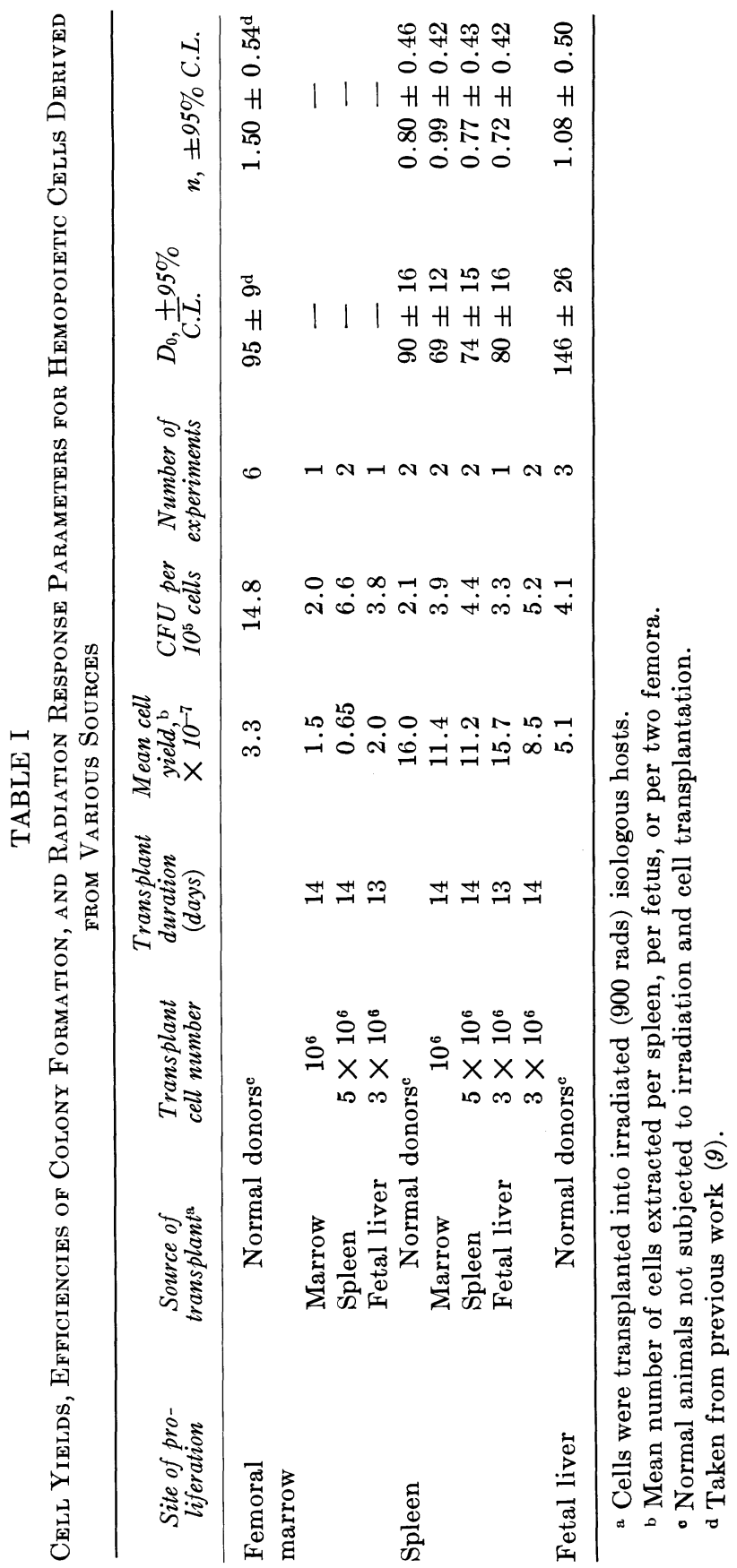


efficiency of these cells was 14.8 colony-forming units (CFU) per $10^{5}$ cells. In contrast, from line 9 of Table I, grafts of cells derived originally from $3 \times 10^{6}$ fetal liver cells, proliferating for 14 days in the spleens of irradiated hosts, yielded an average of $8.5 \times 10^{7}$ cells per spleen, with a colony-forming efficiency of $5.2 \mathrm{CFU}$ per $10^{5}$ cells. In both tables, the efficiencies of colony formation are expressed as colony-forming units $(\mathrm{CFU})$ per $10^{5}$ cells injected, where a $\mathrm{CFU}$ is a cell that reaches the spleen of an irradiated host and there forms a colony. For normal femoral marrow, the fraction of colony-forming cells that reach the spleen has been estimated to be $0.17(7)$.

\section{RESULTS}

Survival Curves for Colony-Forming Cells from Adult Marrow and Fetal Liver

As mentioned above, cells with colony-forming capacity have been demonstrated in normal mouse marrow, spleen, fetal liver, peritoneal fluid, and peripheral blood $(5,6,8)$. Of these sources, the first three yield sufficient numbers of colony-forming cells to permit measurements of radiation survival curves (Table I). In previous studies of the radiation sensitivity of normal mouse hemopoietic cells, normal adult femoral marrow served as the source of cells $(8,9)$. The $\gamma$-ray survival curve for

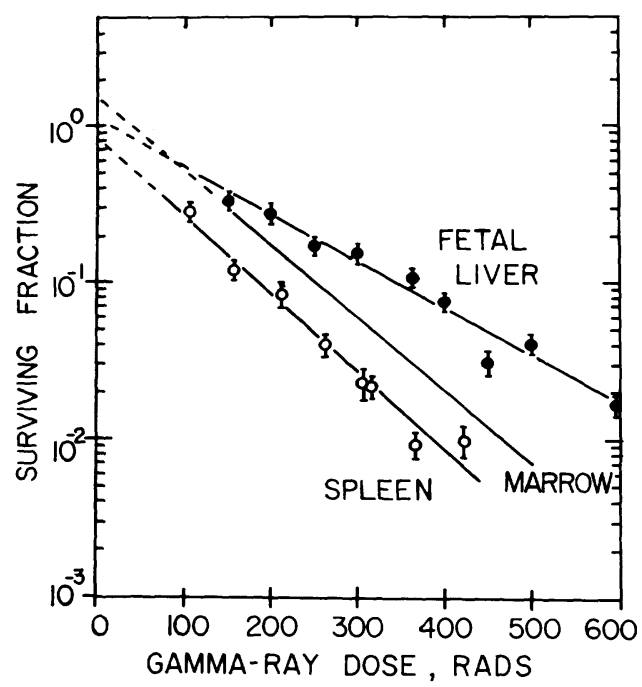

FIg. 1. Survival curves for the colony-forming ability of freshly explanted cells derived from adult femoral marrow, adult spleen, and fetal liver. The standard errors of the colony counts are shown for the individual points. The curve for femoral marrow, shown without points, was taken from previous work $(9)$. The $D_{0}$ values and extrapolation numbers for the curves shown, with their associated $95 \%$ confidence limits, are: for marrow, $95 \pm 9$ rads, $1.50 \pm 0.54$; for spleen, $90 \pm 16 \mathrm{rads}, 0.80 \pm 0.46$; for fetal liver, $146 \pm 26 \mathrm{rads}, 1.08 \pm 0.50$. 
cells from this source has been found to be highly reproducible (9) and therefore provides a suitable reference for survival curves for cells from other sources.

Survival data for the colony-forming ability of cells derived from normal adult spleen and from normal fetal liver are compared with the curve for adult marrow in Fig. 1. It is evident from the figure that the data obtained for fetal liver lie above the curve for adult marrow, and the data for normal spleen lie below the marrow curve. Values for the survival curve parameters, for $D_{0}$, the dose required to reduce the surviving fraction by a factor $1 / e$ on the exponential portion of the survival curve, and for $n$, the extrapolation number (11), were obtained by the method of least squares. These values, with their $95 \%$ confidence limits, are indicated in the figure legend and included in Table I. The $D_{0}$ obtained for fetal liver is significantly greater than those found for adult marrow or spleen. No significant differences in extrapolation number were observed.

\section{Radiation Sensitivity of Transplanted Colony-Forming Cells}

The experimental results presented above indicated that colony-forming cells from fetal liver show a decreased response to $\gamma$-radiation compared with cells from adult bone marrow or spleen. It was of interest to ask whether these differences in sensitivity represented heritable differences between cell types that happened to share the capacity to give rise to spleen colonies. This question could be resolved by comparing colony-forming cells from the various sources, but growing under identical conditions after transplantation into irradiated hosts. The same type of experiment might also be expected to cast light on another possibility, namely that the radiation sensitivity of colony-forming cells depends in part on the anatomical location in which the cells are found. Thus, the sensitivity could be determined for transplanted cells from a given source, proliferating in either spleen or marrow of heavily irradiated recipient mice.

Grafts started from a known number of nucleated cells from bone marrow, spleen, or fetal liver were allowed to proliferate in heavily irradiated mice for 14 days, and at the end of this time cells were harvested from spleen or from femoral marrow; the cell yields and the colony-forming efficiencies of these transplants are given in Table I.

The radiation sensitivity of these cell suspensions was then tested in the usual way (see Materials and Methods). The results are shown in Fig. 2, which show data obtained for grafts derived from marrow, spleen, and fetal liver cells. In each figure, the closed circles represent the results obtained for cells proliferating in the spleens of the irradiated hosts, and the open circles represent results for cells proliferating in femoral marrow. The open triangles are results obtained for cells in spleen colonies, as will be described below. The curves shown without points in Fig. 2 are the survival curves for freshly explanted marrow, spleen, and fetal liver taken from Fig. 1. The values of $D_{0}$ and $n$ for the survival data of Fig. 2 are given in the figure 


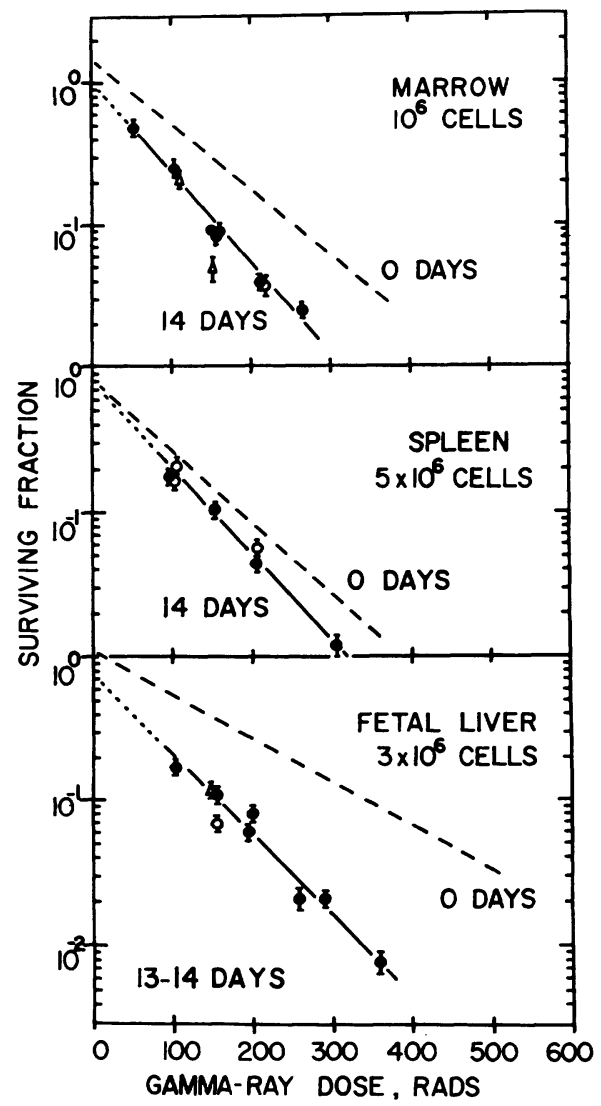

Fig. 2. Survival curves for the colony-forming ability of cells derived from marrow, spleen, or fetal liver transplants after 2 weeks of proliferation in irradiated (900 rads) hosts. Closed circles: cells proliferating in spleen. Open circles: cells proliferating in marrow. Open triangles: cells proliferating in spleen colonies. The dashed curves shown without points were taken from Fig. 1. Standard errors of the colony counts are indicated. The $D_{0}$ values and extrapolation numbers for the solid curves, with their $95 \%$ confidence limits, are: for cells derived from marrow transplants, $69 \pm 12$ rads, $0.99 \pm 0.42$; from spleen, $74 \pm 15$ rads, $0.77 \pm 0.43$; from fetal liver, $80 \pm 16$ rads, $0.72 \pm 0.42$.

legend and in Table I. It is apparent that, after 14 days of proliferation in either spleen or femoral marrow of irradiated hosts, colony-forming cells show a similar radiation sensitivity regardless of their original source. For transplanted cells derived from fetal liver or from adult marrow, the radiosensitivity of cells that had proliferated for 14 days in irradiated hosts was significantly greater than that of freshly explanted cells from these sources. The greatest change was observed for fetal liver, which had the lowest initial radiosensitivity (Fig. 1). 


\section{Radiation Sensitivity of Colony-Forming Cells Derived from Spleen Colonies}

The survival curves presented in Fig. 2 may be interpreted to mean that colonyforming cells proliferating in irradiated hosts have a high and uniform radiation sensitivity only if it can first be shown that the cells tested were derived from the grafts rather than from regenerating endogenous cells derived from the irradiated hosts. The dose of $\mathrm{X}$-radiation given to the hosts in these experiments was 900 rads, which is sufficiently high to render most unlikely the formation of colonies from endogenous cells $(1,12)$. Therefore, cell populations present in spleen colonies could be identified as graft in origin with some assurance. In the experiment, spleen colonies, derived in one case from normal marrow cells and in the other from fetal liver cells injected into irradiated recipients 14 days earlier, were dissected out of the spleens of these animals, pooled, and dispersed to form a cell suspension. The radiation survival of colony-forming cells in this suspension was then measured in the usual way; the results are shown as open triangles in Fig. 2. It is evident that the response of cells derived from individual spleen colonies is not greatly different from the survival curves for cells obtained from the spleens and femora of the irradiated recipients of grafts from various tissues. Thus it appears likely that the cells for which the response to radiation is shown in Fig. 2 were in fact derived in each case from the cellular grafts, and not from regenerating host elements.

\section{Kinetics of the Increase of Sensitivity of Transplanted Fetal Liver Cells}

The uniform radiation sensitivity of transplanted colony-forming cells derived from fetal liver, marrow, and spleen, compared with the widely different results obtained when freshly explanted cells from fetal liver were compared with marrow or spleen, suggested the possibility that the double transplantation procedure used in the experiments might itself be responsible in some way for the uniform sensitivity observed. If fetal liver colony-forming cells twice subjected to the trauma of dispersion and transplantation were found to be more sensitive to radiation than similar cells transplanted only once, then it might be expected that the difference in sensitivity would not increase if time elapsed between the transplantations and, in fact, might even decrease if recovery of transplantation-induced injury occurred. In order to test this possibility, various numbers of cells derived from fetal liver were transplanted into irradiated recipients. At intervals of 2 hours to 14 days thereafter, groups of 10 mice were killed, and the cells obtained from their spleens were tested for response to a single dose (200 rads) of radiation after retransplantation into new recipients. The results of three experiments are given in Table II and Fig. 3. It is clear that the initial low radiation response characteristic of freshly explanted etal liver cells was also observed when cells derived from fetal liver were subjected to two successive transplantations separated in time by only 2 hours, as may be seen by comparing the first two values in the last column of Table II ( $28.6 \%$ and $25.8 \%$ ) with the third value $(28 \%)$. However, the radiation response of these cells had in- 


\section{TABLE II}

Radiation Response, Cell Yields, and Colony-Forming Efficiencies of Transplants of Fetal Liver Cells Multiplying in the Spleens of Irradiated (900 Rads) Hosts

\begin{tabular}{ccccc}
\hline Duration of transplant & $\begin{array}{c}\text { Initial cell number } \\
\text { in transplant }\end{array}$ & $\begin{array}{c}\text { Cell yield, } \\
\times 10^{-7}\end{array}$ & $\begin{array}{c}\text { CFU per } 10^{5} \\
\text { cells }\end{array}$ & $\begin{array}{c}\text { Per cent survival after } \\
200 \text { rads Co }\end{array}$ \\
\hline Normal fetal liver cells $^{\mathrm{a}}$ & & 7.5 & 4.2 & $28.6 \pm 3.8^{\mathrm{b}}$ \\
& & 3.9 & 6.1 & $25.8 \pm 1.8$ \\
2 hours & $3 \times 10^{7}$ & 15.1 & 0.041 & $28 \pm 5.2$ \\
2 days & $3 \times 10^{7}$ & 1.4 & 0.90 & $19 \pm 3.4$ \\
3 days & $10^{7}$ & 1.0 & 1.1 & $12 \pm 1.2$ \\
6 days & $10^{7}$ & 2.8 & 2.5 & $6.4 \pm 1.1$ \\
7 days & $3 \times 10^{6}$ & 1.1 & 2.3 & $3.3 \pm 0.8$ \\
14 days & $3 \times 10^{6}$ & 11.0 & 4.6 & $6.1 \pm 0.6$ \\
& & 6.0 & 5.9 & $8.0 \pm 0.75$ \\
14 days & $10^{7}$ & 14.0 & 3.8 & $8.5 \pm 1.1$ \\
\hline
\end{tabular}

a Derived from normal fetuses not subjected to irradiation or transplantation prior to preparation of cell suspensions.

b Standard error of the mean.

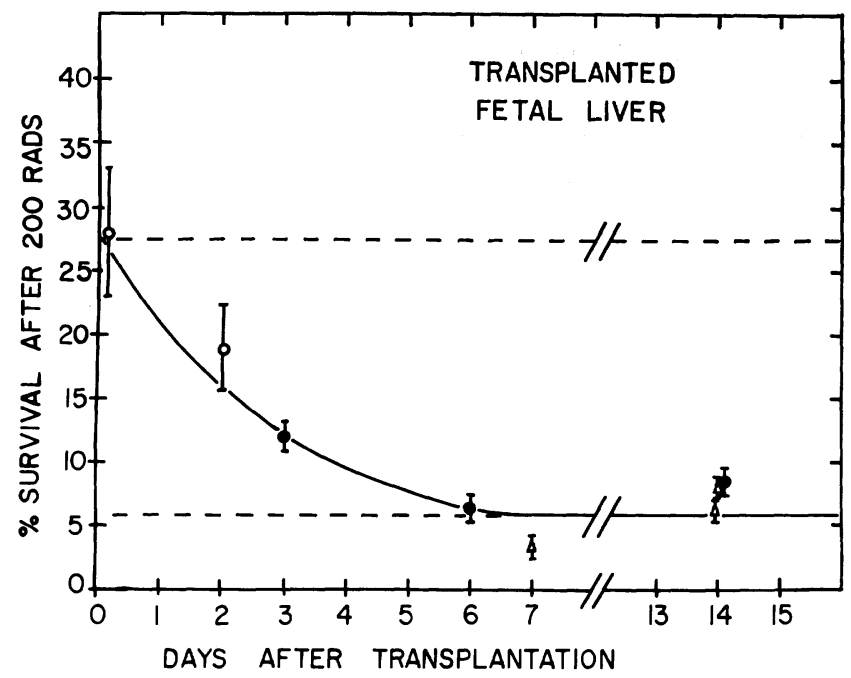

Frg. 3. Kinetics of the increase in response to $200 \mathrm{rads}$ of $\mathrm{Co}^{60} \gamma$-rays, for transplanted cells derived from fetal liver, proliferating in the spleens of irradiated ( 900 rads) hosts. Standard errors of the colony counts are indicated. The different symbols represent transplants containing different numbers of cells: Open circles, $3 \times 10^{7}$ cells; closed circles, $10^{7}$ cells; open triangles, $3 \times 10^{6}$ cells. The upper dashed curve represents the expected response of freshly explanted fetal liver to 200 rads (Fig. 1), the lower dashed curve the expected response of 14-day transplants to 200 rads (Fig. 2). 
creased by 2 days after transplantation, and by 6 days had reached a value similar to that found at 14 days (see also Fig. 2). These results indicate that the increase in radiation sensitivity of cells derived from fetal liver was a consequence of growth of the cells in the irradiated host, rather than of the double transplantation necessary for the test of the radiation sensitivity of transplanted cells.

\section{DISCUSSION}

The experiments reported in this paper demonstrate that hemopoietic colonyforming cells derived from different sources do not exhibit a uniform sensitivity to radiation. In particular, colony-forming cells derived from fetal liver were found to show a decreased response to radiation when compared with cells derived from adult femoral marrow or spleen. It was also found that a rapid increase in the radiosensitivity of cells derived from fetal liver occurred during the first few days after transplantation of these cells into irradiated hosts (Table II and Fig. 3), and a similar shift probably occurred for transplanted marrow (Fig. 2). The possibility that the increase might be the result of selection of a minority cell type with relatively high radiosensitivity is rendered unlikely by the rapidity of the change in radiosensitivity of fetal liver cells after transplantation. A more likely interpretation of the results is that the properties of colony-forming cells are not uniform and that the change in radiosensitivity represents a change in the properties of colony-forming cells occurring with transplantation. This concept is in agreement with the findings of a previous study in which the capacity for self-renewal of freshly explanted colony-forming cells from marrow (4) or from fetal liver (13) was compared to that of colony-forming cells derived from spleen colonies. These experiments showed that the proliferative capacity of the progeny colony-forming cells found in spleen colonies was less than that of their progenitors, found in marrow or fetal liver. Both the loss of proliferative capacity and the increased response to radiation occurring with proliferation support the view that the process of self-renewal, by which a colony-forming cell gives rise to more cells with colony-forming potential, is not an exact one, but rather that important differences in properties exist between progenitors and descendants.

The changes in the properties of colony-forming cells observed in our experiments occurred under the artificial conditions of cell transplantation and may not be relevant to the normal physiological situation. However, the differences in radiation sensitivity found between colony-forming cells derived from fetal liver and those from adult hemopoietic organs suggest that similar changes may occur under normal conditions; the liver is the site of blood formation in embryo, whereas this function is situated in marrow and spleen in adult life. One may assume that the colony-forming cells of fetal liver are progenitors of the colony-forming cells of adult tissues. If this assumption is correct, the differences in $D_{0}$ found between cells from fetal sources and those from adult sources may have a similar basis to the changes found after transplantation of cells from fetal liver. 
It could be argued that the transition from the relatively less radiosensitive state of freshly explanted cells from fetal liver to the relatively more sensitive state of transplanted cells derived from the same source was due entirely to the effects of the transplantation procedure, and not to the subsequent cellular proliferation. However, the observation that this transition occurred over a period of 6 days, rather than abruptly, suggests that the transplantation procedure employed is not the whole explanation of the change. Nevertheless, some role of the transplantation procedure cannot be ruled out, since, in previous experiments, no change in the radiation sensitivity of cells derived from marrow was observed when they were irradiated 5 days after a single transplantation into irradiated hosts (10). This apparent discrepancy may be related in some way to the total number of cells present in the hosts at the time of irradiation of the colony-forming cells, since the results of preliminary experiments have indicated that the rate of change of the radiation sensitivity of transplanted cells derived from marrow depends on the number of cells in the transplant.

The variation in radiation sensitivity observed for colony-forming cells may be important in relation to our understanding of the cellular basis of animal survival after irradiation. On the assumption that colony-forming cells may be identified with at least some of the stem cells on which vital repopulation of radiation-damaged hemopoietic tissue depends, it would be possible to construct a rather simple model of the effects of total-body irradiation on hemopoietic tissue by making the further assumption that populations of colony-forming cells possess a relatively uniform radiation sensitivity, regardless of their site or rate of proliferation. Unfortunately, the results presented in this paper indicate that the latter simplifying assumption would be incorrect, in that the radiation sensitivity of colony-forming cells may vary by a factor of about 2 , depending on the previous history of the cells.

In the light of these findings, the results of a previous study (1) require reappraisal. In it, the kinetics of proliferation of endogenous colony-forming cells surviving in the spleens of sublethally (400 rads) irradiated mice were examined. When it was assumed that endogenous spleen colony-forming cells may he characterized by a $D_{0}$ dose of 95 rads, a value of 32 hours was obtained for the doubling time of these cells. If the actual $D_{0}$ for cells multiplying in spleen is less than this value (Table I), an estimate is obtained for the doubling time of colony-forming cells in spleen which is in agreement with the doubling time of 20 to 25 hours reported in a later study (10).

One may also ask whether the increase in the radiosensitivity of colony-forming cells with time after transplantation is a reversible effect. The results of preliminary experiments for cells derived from marrow indicate that it is, but that the return to normal radiosensitivity occurs gradually, over a period of several months. In the interval prior to completion of this process, the hemopoietic tissues of the irradiated animal, although repopulated with cells, should appear unusually sensitive to radiation. It is possible that the irreparable injury reported by a number of workers 
(14) may be related to this phenomenon. However, it has yet to be demonstrated that these effects, observed in transplanted cells, also occur after sublethal totalbody irradiation in intact animals.

\section{SUMMARY}

1. Hemopoietic colony-forming cells derived from adult femoral marrow, adult spleen, and fetal liver did not exhibit a uniform sensitivity to radiation when freshly explanted cells from these sources were tested in the same way for the survival of colony-forming ability after irradiation in vivo. The $D_{0}$ values and extrapolation numbers observed were: for adult femoral marrow, $95 \pm 9 \mathrm{rads}, 1.50 \pm 0.54$; for adult spleen, $90 \pm 16 \mathrm{rads}, 0.80 \pm 0.46$; for fetal liver, $146 \pm 26 \mathrm{rads}, 1.08 \pm$ 0.50 .

2. Fourteen days after transplantation into X-irradiated (900 rads) hosts, hemopoietic colony-forming cells derived from femoral marrow, spleen, or fetal liver showed a relatively uniform, increased radiation sensitivity for cells proliferating in spleen or in marrow. The $D_{0}$ values and extrapolation numbers for cells proliferating in spleen were found to be: for cells derived originally from marrow, $69 \pm 12$ rads, $0.99 \pm 0.42$ for cells derived from spleen, $74 \pm 15 \mathrm{rads}, 0.77 \pm 0.43$; for cells derived from fetal liver, $80 \pm 16$ rads, $0.72 \pm 0.42$.

3 . For cells derived from fetal liver, the increase in radiation response took place during the first 6 days after transplantation of the cells into irradiated hosts.

\section{ACKNOWLEDGMENTS}

We are grateful for the excellent technical assistance of Miss Rosemary Wyncoll and Messrs. R. Course, P. Csordas, A. Galberg, J. Hicks, R. Kuba, and F. Mik.

ReCeIved: August 10, 1964

\section{REFERENCES}

1. J. E. Till and E. A. McCulloch, Repair processes in irradiated mouse hematopoietic tissue. Ann. N.Y. Acad. Sci. 114, 115-125 (1964).

2. E. A. McCulloch, L. Siminovitch, and J. E. Till, Spleen-colony formation in anemic mice of genotype $W W^{v}$. Science 144, 844-846 (1964).

3. G. Cudkowicz, M. Bennett, and G. M. Shearer, Pluripotent stem cell function of the mouse marrow "lymphocyte." Science 144, 866-868 (1964).

4. L. Siminovitch, J. E. Trle, and E. A. McCulloch, Decline in colony-forming ability of marrow cells subjected to serial transplantation into irradiated mice. J. Cellular Comp. Physiol. 64, 23-31 (1964).

5. E. A. McCulloch and J. E. Till, Repression of colony-forming ability of C57BL hematopoietic cells transplanted into non-isologous hosts. J. Cellular Comp. Physiol. 61, 301308 (1963).

6. L. J. Cole, Hemopoietic restoration in lethally X-irradiated mice injected with peritoneal cells. Am. J. Physiol. 204, 265-267 (1963).

7. L. Siminovitch, E. A. McCulloch, and J. E. Till, The distribution of colony-forming cells among spleen colonies. J. Cellular Comp. Physiol. 62, 327-336 (1963). 
8. J. E. Till and E. A. McCulloch, A direct measurement of the radiation sensitivity of normal mouse bone marrow cells. Radiation Res. 14, 213-222 (1961).

9. E. A. McCulloch and J. E. Till, The sensitivity of cells from normal mouse bone marrow to gamma radiation in vitro and in vivo. Radiation Res. 16, 822-832 (1962).

10. E. A. McCulloch and J. E. Till, Proliferation of hemopoietic colony-forming cells transplanted into irradiated mice. Radiation Res. 22, 383-397 (1964).

11. T. Alper, N. E. Gillies, and M. M. Elkind, The sigmoid survival curve in radiobiology. Nature 186, 1062-1063 (1960).

12. J. E. Till and E. A. McCulloch, Early repair processes in marrow cells irradiated and proliferating in vivo. Radiation Res. 18, 96-105 (1963).

13. J. E. Till, E. A. McCulloch, and L. Siminovitch, The isolation of variant cell lines during serial transplantation of hemopoietic cells derived from fetal liver. J. Natl. Cancer Inst. 33, 707-720 (1964).

14. G. A. SACHER, Reparable and irreparable injury: A survey of the position in experiment and theory. In Radiation Biology and Medicine (W. D. Claus, ed.), pp. 283-313, AddisonWesley Publishing Company, Reading, Massachusetts, 1958. 